\title{
Ergonomic assessment of neck posture in the minimally invasive surgery suite during laparoscopic cholecystectomy
}

\author{
M. J. van Det · W. J. H. J. Meijerink • \\ C. Hoff · M. A. van Veelen · J. P. E. N. Pierie
}

Received: 21 November 2007 / Accepted: 4 June 2008/Published online: 12 July 2008

(C) Springer Science+Business Media, LLC 2008

\begin{abstract}
Background With the expanding implementation of minimally invasive surgery, the operating team is confronted with challenges in the field of ergonomics. Visual feedback is derived from a monitor placed outside the operating field. This crossover trial was conducted to evaluate and compare neck posture in relation to monitor position in a dedicated minimally invasive surgery (MIS) suite and a conventional operating room.

Methods Assessment of the neck was conducted for 16 surgeons, assisting surgeons, and scrub nurses performing a laparoscopic cholecystectomy in both types of operating room. Flexion and rotation of the cervical spine were measured intraoperatively using a video analysis system. A two-question visual analog scale (VAS) questionnaire was used to evaluate posture in relation to the monitor position. Results Neck rotation was significantly reduced in the MIS suite for the surgeon $(p=0.018)$ and the assisting surgeon $(p<0.001)$. Neck flexion was significantly improved in the MIS suite for the surgeon $(p<0.001)$ and the scrub nurse $(p=0.018)$. On the questionnaire, the operating room team scored their posture significantly
\end{abstract}

M. J. van Det $(\bowtie)$ C. Hoff · J. P. E. N. Pierie

Department of Surgery, Leeuwarden Medical Center, P.O. Box 888, 8901 BR Leeuwarden, The Netherlands

e-mail: marc.van.det@znb.nl

W. J. H. J. Meijerink

Department of Gastro Intestinal Surgery, Free University

Medical Center, Amsterdam, The Netherlands

M. A. van Veelen

Faculty of Industrial Design Engineering, Delft University

of Technology, Delft, The Netherlands higher in the MIS suite and also indicated fewer musculoskeletal complaints.

Conclusions The ergonomic quality of the neck posture is significantly improved in the MIS suite for the entire operating room team.

Keywords Ergonomics - Laparoscopic cholecystectomy · Minimally invasive surgery $\cdot$ Neck · Operating room .

Posture

Minimally invasive surgery (MIS) plays a major part in modern abdominal surgery, urology, and gynecology and has become the treatment of choice for a still growing number of procedures. Most of the advantages with MIS are patient related. Less blood loss, less postoperative pain, shorter hospital admissions, quicker reintroduction into society, and a superior cosmetic result are some wellestablished MIS advantages [1-4].

On the other hand, MIS confronts the surgeon and his or her team with some challenging aspects, primarily in the area of ergonomics and efficiency [5, 6]. The necessity of additional equipment-including electrocautery and insufflation devices, monitors, video equipment, wiring, and tubing, usually stored outside the operating room on large heavy trolleys-has compromised operating room efficiency and prolonged turnover times [7].

During the procedure, the surgeon must work with long instruments that move invertedly inside the abdomen and with a certain scaling effect, also known as the fulcrum effect [8]. The entire operating team derives the visual feedback of their actions from a monitor positioned on top of a laparoscopic trolley that stands outside the operative field and away from the patient. Due to this positioning, the 
line of vision is diverted away from the line of action, creating an awkward posture including rotation of the spine, extension of the neck, and elevation of the upper extremities. This causes musculoskeletal complaints and possibly compromises surgical task performance [9-12].

Many different solutions have been devised to overcome these various drawbacks of MIS. The most versatile and most achievable solution is the dedicated minimally invasive surgery (MIS) suite [7, 13, 14]. These fully integrated operating rooms are equipped with permanently installed laparoscopic equipment that is operational on demand inside the operating room. This equipment, together with multiple flat-screen monitors, is attached to a ceilingmounted suspension system to facilitate versatile positioning around the operative field. The increased freedom of monitor positioning should, when used correctly, provide an improved ergonomic posture for the entire operating team and prevent extreme head and neck angulations in the axial and sagittal plane.

This study compared the ergonomic posture of the cervical spine for the entire operating room team during laparoscopic cholecystectomy performed in the traditional operating room with a cathode ray tube (CRT) monitor on top of a laparoscopic trolley and in the MIS suite with flatscreen monitors suspended from the ceiling. We hypothesized that there would be a significant improvement in neck posture for the entire team in the MIS suite, resulting in a better ergonomic work environment and a reduction in posture-related musculoskeletal complaints.

\section{Materials and methods}

Study design

The study was performed in the Department of Surgery at the Leeuwarden Medical Center (MCL). In a clinical setting, we analyzed the posture of the cervical spine of the surgeon, the assisting surgeon, and the scrub nurse during laparoscopic cholecystectomies for patients with symptomatic cholecystolithiasis. For this study, 16 surgeons, 16 assisting surgeons, and 16 scrub nurses were randomly assigned to perform their tasks during a laparoscopic cholecystectomy in a traditional operating room or MIS suite.

After assessment of the first operation, those assigned to the MIS suite performed their second cholecystectomy in a traditional operating room and vice versa, allowing each participant to be his own matched control. Flexion and rotation of the cervical spine was monitored with a video analysis system for 5 min during the dissection of Calot's triangle. During this stage of the cholecystectomy, the tasks of the operating room team and their position around the table is submitted to little variance between different cases, and the entire team is focusing on the monitors. Therefore, the operating room team's neck posture is completely dependant on the monitor position in relation to the operating table.

In the traditional operating room, the laparoscopic equipment is installed on a movable trolley. On the top of this trolley, one CRT monitor is installed on a rotating platform. This 19-in. monitor is not height adjustable, and the center of the screen is elevated $165 \mathrm{~cm}$ from the floor. For a laparoscopic cholecystectomy, we use a single trolley with one CRT monitor positioned at the right top end of the table, opposite the surgeon, who stands on the left side of the table (Fig. 1). On the left side of the surgeon stands the scrub nurse. She prepares the instruments and operates the camera. An assisting surgeon stands on the right side of the table. He controls a grasping forceps that provides traction on the gallbladder for visualization of the operative field.

In the MIS suite, the operating room team has similar positions around the table, and all three available monitors are used (Fig. 2). A dual flat panel is positioned on the right side of the table opposite the operator and the scrub nurse. A third flat screen is positioned on the left side of the table for the assisting surgeon. Screen height, distance, and inclination can be adjusted to the preference of the observers.
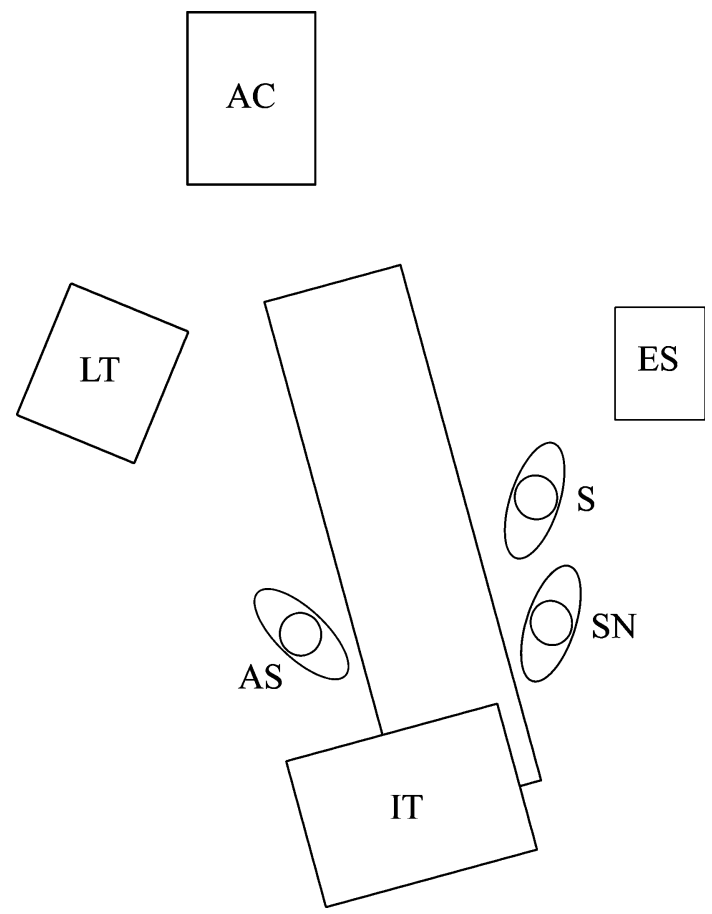

Fig. 1 Operative setup in the conventional operating room. AC, anesthesia console; LT, laparoscopy trolley; ES, electrocautery and suction devices; S, surgeon; AS, assisting surgeon; SN, scrub nurse; IT, instrument table 


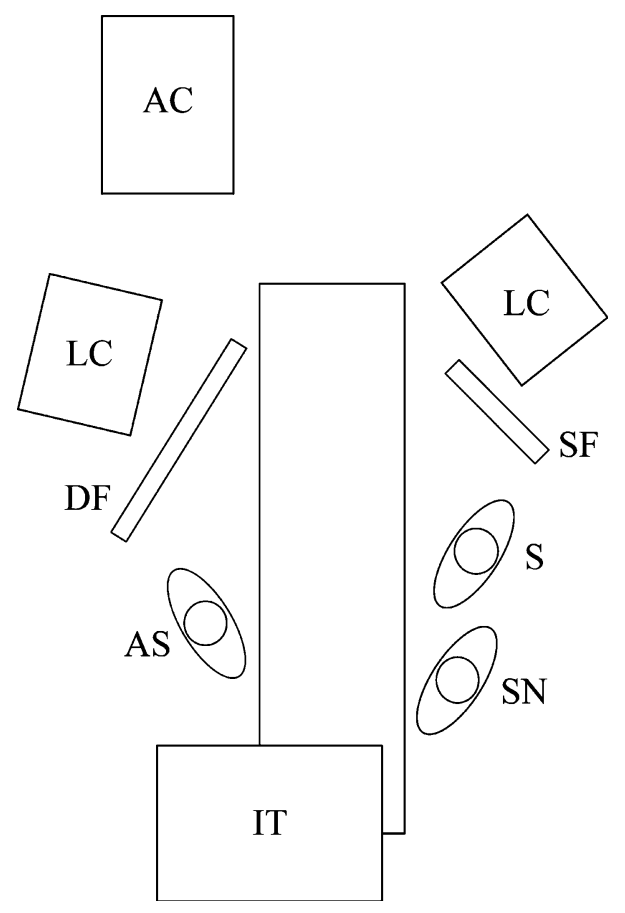

Fig. 2 Operative setup in the minimally invasive surgery (MIS) suite. AC, anesthesia console; LC, laparoscopic consoles on pneumatic booms; DF, double flat screen; SF, single flat screen; S, surgeon; AS, assisting surgeon; SN, scrub nurse; IT, instrument table

For the surgeon, the screen is ideally placed right in front near his hands and instruments to permit a moderate downward viewing angle of $10^{\circ}$ to $30^{\circ}$ without axial rotation $[10,15,16]$. For the assisting surgeon and the scrub nurse, the screen is ideally placed just below eye level to allow a slightly downward viewing direction of $0^{\circ}$ to $15^{\circ}$. Upward viewing angles that cause harmful neck extension are to be avoided.

For all the participants, minimal neck torsion was aspired. An axial rotation less than $15^{\circ}$ was considered ergonomically acceptable.

Body posture assessment was performed with a video analysis system consisting of two digital cameras mounted on a standard and connected to a laptop computer. One camera was positioned above the participant for observation of the axial rotation of the head compared with the trunk. The second camera was positioned perpendicular to the participant's viewing direction for observation of the head's flexion in the sagittal plane. The cameras took pictures simultaneously every $2 \mathrm{~s}$ for a period of $5 \mathrm{~min}$ per participant ( 150 photos per camera per participant).

After each procedure, every participant was asked to fill in a questionnaire containing two questions that had to be answered on a 100-mm visual analog scale (VAS). The first question asked the participant to judge the ergonomic quality of his or her posture in relation to the monitor position on a scale of 0 (very bad) to 100 (optimal). The second question asked the participant to indicate whether any musculoskeletal complaints were experienced as a result of his or her posture on a scale of 0 (no complaints) to 100 (disabling complaints).

Data analysis

Measurements on the photos were digitally performed with a line-angle measuring tool in Adobe Photoshop 9.0 (Adobe Systems Incorporated, San Jose, CA, USA). To facilitate accurate measurement in the horizontal plane (rotation), we attached markers on the surgical hat and on the acromion of each shoulder. For measurements in the sagittal plane, we used the anatomic ear-eye line (EEL) running through the tragus of the ear and the canthus of the eye (Fig. 3A). In neutral position of the head and neck, the EEL has an inclined angle of approximately $15^{\circ}$ to the horizontal [16].
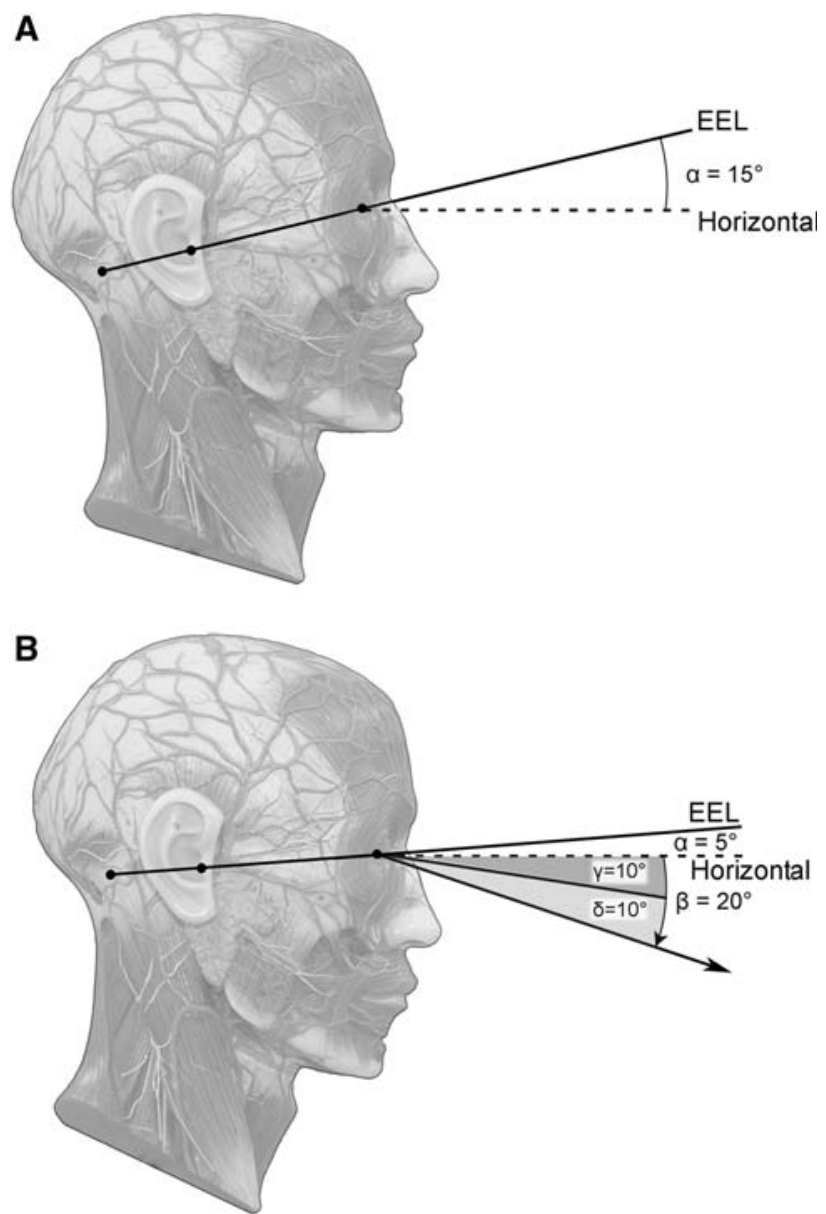

Fig. 3 Flexion of the head and viewing direction. (A) Neutral position. The anatomic ear-eye line (EEL) is $15^{\circ}$ to the horizontal. (B) Head in $10^{\circ}$ flexion. The anatomic EEL is now measured at $5^{\circ}$ above the horizontal. The viewing direction (arrow) at angle $\beta$ is a combined effort of head flexion $\gamma$ and gaze angle $\delta$ of the eyes 
We also recorded eye height and screen height in relation to the floor and the viewing distance from eye to monitor to enable calculation of the viewing direction (viewing direction $=$ Sin [eye height - screen height] $/$ viewing distance]). The viewing direction is a combined effort of neck flexion/extension and angle of gaze performed by the extraocular musculature (Fig. 3B).

\section{Statistical analysis}

A power analysis was performed to calculate sample size for paired analysis. To find a $5^{\circ}$ difference in flexion and rotation between the groups with a standard deviation of $10^{\circ}$, sample sizes of at least 10 participants are needed, with a type 1 error rate set at 0.05 and power set at 0.80 . Because the desired difference and standard deviations were estimates, we performed 16 measurements in each group to avoid type 1 and type 2 errors.

Continuous variables were compared with the Student $t$ test for paired observations and are presented as mean \pm standard deviation. Effect sizes were calculated only for the statistically significant results because differences between the results for the two types of operating room due to sample fluctuation have no clinical relevance. Cohen's effect size $d$ for related samples was used to estimate the magnitude of the difference between the results for the MIS suite and those for the conventional operating room.

To avoid overestimation of the effect with Cohen's thresholds, mean differences were standardized by the pooled standard deviation [17]. According to these thresholds, an effect size less than 0.20 indicates a trivial difference, 0.20 to 0.50 a small difference, 0.50 to 0.80 a moderate difference, and 0.80 or more a large difference [18]. Middel et al. [19] showed that effect size reflects clinical relevance.

In the current study, an effect size of 0.50 or larger was considered to be a clinically relevant difference between groups. All statistical tests were two-tailed. A $p$ value less than 0.05 was used for all tests to indicate statistical significance. All statistical analyses were performed using SPSS 13.0.1 for Windows (SPSS, Chicago, IL, USA).

\section{Results}

The neck flexion and rotation of all 48 subjects were successfully analyzed for both the MIS suite and the traditional operating room. Of the 16 teams, 9 (54\%) started in the MIS suite. The remainder started in the conventional operating room. All laparoscopic cholecystectomies were completed without any adverse events.
Table 1 Mean neck rotation of the participants ${ }^{\mathrm{a}}$

\begin{tabular}{llcrr}
\hline & \multicolumn{2}{l}{ Rotation degrees } & $p$-value & ES \\
\cline { 2 - 3 } & MIS suite & Conventional OR & & \\
\hline Surgeon & $5.91 \pm 4.6$ & $11.11 \pm 7.5$ & 0.018 & 0.83 \\
Assisting surgeon & $9.85 \pm 10$ & $38.77 \pm 14$ & $<0.001$ & 2.37 \\
Scrub nurse & $8.95 \pm 7.7$ & $1.08 \pm 6.2$ & 0.461 & \\
\hline
\end{tabular}

MIS, minimally invasive surgery; OR, operating room; ES, effect size

a The target value for mean rotation was less than 15 degrees

Table 1 shows the results for rotation. A statistically significant reduction in neck rotation was achieved in the MIS suite for the surgeon $\left(5.2^{\circ} ; p=0.018\right)$ and the assisting surgeon $\left(29.2^{\circ} ; p<0.001\right)$. Effect sizes indicated large differences between the performances in the MIS suite and the conventional operating room. The scrub nurse did not significantly reduce her neck rotation in the MIS suite.

Table 2 shows the results for flexion, gaze angle, and viewing direction. Neck flexion was significantly improved for the surgeon in the MIS suite, preventing extension of the neck. Paired comparisons for the participating surgeons showed that flexion was increased by $7.2^{\circ}$ on the average $(p<0.001)$. Also, the viewing direction significantly declined $(p<0.001)$. The angle of gaze, accounted for by

Table 2 Mean neck flexion, viewing direction, and gaze angle ${ }^{\mathrm{a}}$

\begin{tabular}{|c|c|c|c|c|}
\hline & \multicolumn{2}{|c|}{ Angle in degrees } & \multirow[t]{2}{*}{$p$-value } & \multirow[t]{2}{*}{$\mathrm{ES}$} \\
\hline & MIS suite & $\begin{array}{l}\text { Conventional } \\
\text { OR }\end{array}$ & & \\
\hline \multicolumn{5}{|l|}{ Surgeon } \\
\hline Flexion & $-3.52 \pm 3.2$ & $3.70 \pm 2.9$ & $<0.001$ & 2.37 \\
\hline $\begin{array}{l}\text { Viewing } \\
\text { direction }\end{array}$ & $-12.87 \pm 4.3$ & $-4.13 \pm 2.6$ & $<0.001$ & 2.46 \\
\hline Gaze angle & $-9.35 \pm 4.2$ & $-7.83 \pm 7.4$ & 0.083 & 0.25 \\
\hline \multicolumn{5}{|c|}{ Assisting surgeon } \\
\hline Flexion & $5.43 \pm 7.1$ & $6.64 \pm 6.4$ & 0.397 & \\
\hline $\begin{array}{l}\text { Viewing } \\
\text { direction }\end{array}$ & $-0.23 \pm 4.8$ & $-2.02 \pm 4.7$ & 0.105 & \\
\hline Gaze angle & $-5.67 \pm 5.8$ & $-8.67 \pm 4.8$ & 0.039 & 0.56 \\
\hline \multicolumn{5}{|l|}{ Scrub nurse } \\
\hline Flexion & $0.40 \pm 5.2$ & $7.96 \pm 4.8)$ & $<0.001$ & 1.51 \\
\hline $\begin{array}{l}\text { Viewing } \\
\text { direction }\end{array}$ & $-8.17 \pm 7.1$ & $-1.20 \pm 2.6$ & 0.002 & 1.30 \\
\hline Gaze angle & $-9.86 \pm 6.9$ & $-9.16 \pm 4.2$ & 0.828 & \\
\hline
\end{tabular}

MIS, minimally invasive surgery; OR, operating room; ES, effect size

a The target values for neck flexion were neutral to slightly inclined for the surgeon and neutral for the assisting surgeon and the scrub nurse. The target values for viewing direction were $-10^{\circ}$ to $-30^{\circ}$ for the surgeon and $0^{\circ}$ to $-15^{\circ}$ for the assisting surgeon and the scrub nurse 
Table 3 Two-question visual analog scale (VAS) questionnaire (0$100 \mathrm{~mm})^{\mathrm{a}}$

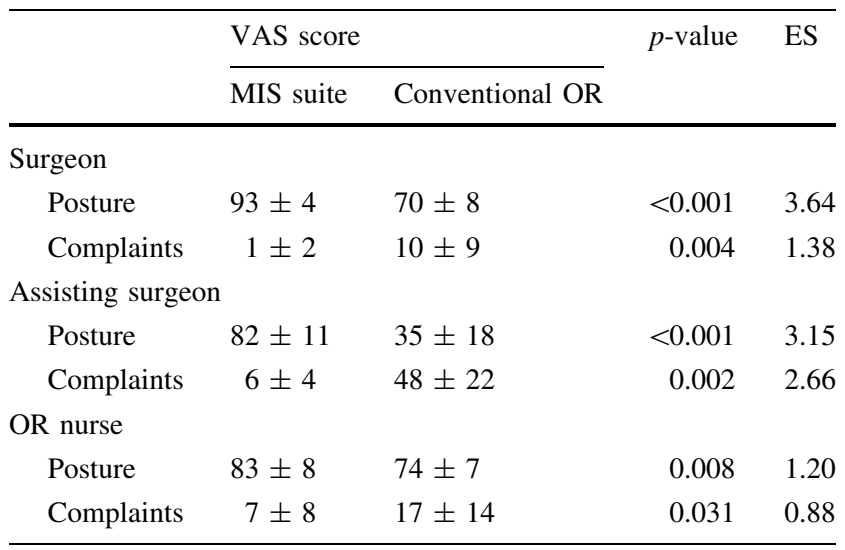

MIS, minimally invasive surgery; OR, operating room; ES, effect size

${ }^{a}$ Mean scores measured from a 100-mm VAS: 100 (optimal posture) to 0 (no complaints)

the extraocular muscles, was not influenced by monitor position $(p=0.64)$.

The assisting surgeon did not improve neck flexion or viewing direction in the sagittal plane. The scrub nurse improved neck flexion significantly in the MIS suite, preventing extension of the neck. Paired comparisons for the participating scrub nurses showed that flexion was increased by $7.3^{\circ}$ on the average $(p=0.018)$. Also, the viewing direction significantly declined $(p<0.001)$. The angle of gaze was not influenced by monitor position $(p=0.82)$. For the surgeon and the scrub nurse, effect sizes indicated large differences in the performances for flexion and viewing direction between the MIS suite and the conventional operating room.

Table 3 shows the results of the questionnaire. On the VAS, the surgeons rated the ergonomic quality of their posture $23 \mathrm{~mm}$ more positive in the MIS suite $(p<0.001)$. In the second question, they indicated that they did not experience many complaints in either type of operating room. The questionnaire suggested a slight but statistically significant reduction of musculoskeletal complaints in the MIS suite. The assisting surgeons scored their posture $47 \mathrm{~mm}$ more positive in the MIS suite $(p=0.001)$ and also indicated experiencing substantially fewer musculoskeletal complaints in the MIS suite $(42 \mathrm{~mm} ; p=0.002)$. The scrub nurses scored their posture $9 \mathrm{~mm}$ better in the MIS suite $(p=0.008)$ and suggested that they experienced a minimal but statistically significant reduction in musculoskeletal complaints $(10 \mathrm{~mm} ; p=0.031)$.

\section{Discussion}

The literature provides evidence that physical discomfort during MIS is very common [6, 9]. Complaints concerning the neck and back can be caused by an uncomfortable and static posture in relation to the position of the monitor. Studies examining the most comfortable posture advise a viewing direction straight ahead and at a slightly downward angle $[9,12,20]$. In this monitor configuration, the working posture is the most neutral. Studies examining efficiency of movement and task performance during MIS advise a monitor configuration straight in front of the surgeon and in the direct vicinity of the operating field [10, $15,21,22]$. This monitor configuration brings the viewing direction back to the direction of work and restores the natural eye-hand-target axis. Optimal monitor positioning is a balance between these two entities to create a work environment that enables the operating team to work efficiently for long periods without experiencing physical discomfort. This may save valuable operating room time and reduce physical overexertion.

To date, we have demonstrated only postural improvement of the cervical spine. Our video analysis system proved to be an accurate and noninvasive means of measuring multiple persons during one procedure at a high frequency in a sterile environment. Most complaints related to the monitor position involve the cervical spine and the upper extremities. However, when multiple working directions are adapted during the procedure, it might be interesting to observe the entire spine.

Because the operating room teams performed only their normal tasks during the procedure in both operating rooms, no order effects were expected between the first and the second measurements. For this reason, the current study was very suitable for a crossover design. With this study design, we could eliminate the possibility of covariate imbalances between the study groups, which is very important in posture analysis.

In this study, we chose to observe laparoscopic cholecystectomy because this is a frequently performed and relatively short procedure that has standard and clearly identifiable stages and requires only one working direction. For this procedure, we demonstrated significant ergonomic benefits of the MIS suite. We expect that these benefits will increase with increasing duration and complexity of the procedure, especially when the surgeon has to work in multiple directions.

In the conventional operating room, we used a single monitor setup for a laparoscopic cholecystectomy because this is the daily practice at MCL. If a second monitor had been used, the neck rotation of the assisting surgeon would have been decreased, with neck extension remaining the same or even increased because our accessory monitor sits on a tall trolley elevating the center of the screen to $177 \mathrm{~cm}$ compared with $165 \mathrm{~cm}$ using the main trolley.

We used a dedicated MIS suite to demonstrate the ergonomic benefit of monitors connected to a ceiling-mounted 
suspension system. This feature is not exclusive to the MIS suite concept, but also can be found with other configurations. Many other simpler solutions can be applied to improve the monitor position. Accessory laparoscopic trolleys and small height-adjustable flat screens attached to the side of the trolley could achieve the same ergonomic advantage. However, during more complex procedures that require working in multiple directions, the entire trolley still must be moved to maintain a neutral viewing direction.

The ergonomic benefit of suspended monitors is not the only consideration for hospitals building an MIS suite. Safety, efficiency, and financial aspects also are important and can be improved for a clinician working in an MIS suite. Improved efficiency can reduce expensive operating room time and allow planning of extra procedures. Efficiency is improved because the laparoscopic equipment is operational on demand inside the operating room. This reduces preparation time and may prevent time loss caused by connection errors. During operations, the operating room team can work more efficiently because of improved ergonomics. The laparoscopic equipment is remote controlled by the circulating nurse from her nursing station or by the surgeon using a touch panel or voice control. This may allow a reduction of personnel in the operating room. Safety is improved by reducing connection errors with the permanently installed equipment, and because the equipment is remote controlled, the circulating nurse does not have to approach the sterile field as often. Equipment such as video documentation devices, not needed directly for the patient, can be moved away from the operating field. The power supply, network and audiovisual connections, and supply of gases are delivered through the ceiling-mounted power beams to the equipment from sources outside the operating room. These features reduce the number of hazardous cables and tubes running across the floor. They create more free space in the operating room and contribute to a safe and efficient work environment.

A new MIS suite does not automatically ensure that the ergonomic posture will be improved without special attention to this aspect. The most important condition for improved ergonomics is an entire operating room staff with some knowledge of ergonomics and the probable causes of posture-related complaints during MIS. Second, the operating room staff must be aware of the possible solutions to these complaints and the way these ergonomic solutions can be achieved in the MIS suite. This requires additional training in ergonomics for everyone working with MIS and technical instructions for everyone using the MIS suite.

Because of the versatile monitor positioning in the MIS suite, a screen may be positioned incorrectly just as easily. Inadequate use of the MIS suite was excluded in this study by optimizing monitor positions for both types of operating room during each procedure.
In conclusion, this study demonstrates that operating rooms with suspended monitors, as in modern MIS suites, can improve the posture of the entire operating room team significantly. Using suspended monitors, the surgeon, the assisting surgeon, and the scrub nurse can stand straight in front of a monitor with minimal neck rotation. With an inclined monitor position, the surgeon's viewing direction and working direction are brought together again, which will enhance his operating performance and efficiency. For the assisting surgeon and the scrub nurse, the monitors can be adjusted to avoid neck extension and rotation.

Acknowledgments We thank J. M. P. Collins for reviewing the text, B. Middel for advising on statistical methods, and the MCL Department of Medical Photography for creating the figures.

\section{References}

1. Berggren U, Gordh T, Grama D, Haglund U, Rastad J, Arvidsson D (1994) Laparoscopic versus open cholecystectomy: hospitalization, sick leave, analgesia, and trauma responses. Br J Surg 81:1362-1365

2. Hendolin HI, Paakonen ME, Alhava EM, Tarvainen R, Kemppinen T, Lahtinen P (2000) Laparoscopic or open cholecystectomy: a prospective randomised trial to compare postoperative pain, pulmonary function, and stress response. Eur J Surg 166:394-399

3. Richards C, Edwards J, Culver D, Emori TG, Tolson J, Gaynes R (2003) Does using a laparoscopic approach to cholecystectomy decrease the risk of surgical site infection? Ann Surg 237:358-362

4. Schellekens PC, Bijnen AB, Honing M, Lourens J, de Ruiter P (1995) Results of the introduction of laparoscopic cholecystectomy on morbidity and mortality of gallbladder surgery in a large regional hospital. Ned Tijdschr Geneeskd 139:723-727

5. Berguer R (1999) Surgery and ergonomics. Arch Surg 134: 1011-1016

6. Van Veelen MA, Nederlof EA, Goossens RH, Schot CJ, Jakimowicz JJ (2003) Ergonomic problems encountered by the medical team related to products used for minimally invasive surgery. Surg Endosc 17:1077-1081

7. Kenyon TA, Urbach DR, Speer JB, Waterman-Hukari B, Foraker GF, Hansen PD, Swanstrom LL (2001) Dedicated minimally invasive surgery suites increase operating room efficiency. Surg Endosc 15:1140-1143

8. Gallagher AG, McClure N, McGuigan J, Ritchie K, Sheehy NP (1998) An ergonomic analysis of the fulcrum effect in the acquisition of endoscopic skills. Endoscopy 30:617-620

9. Berguer R, Forkey DL, Smith WD (1999) Ergonomic problems associated with laparoscopic surgery. Surg Endosc 13:466-468

10. Erfanian K, Luks FI, Kurkchubasche AG, Wesselhoeft CW Jr, Tracy TF Jr (2003) In-line image projection accelerates task performance in laparoscopic appendectomy. J Pediatr Surg 38:1059-1062

11. Hemal AK, Srinivas M, Charles AR (2001) Ergonomic problems associated with laparoscopy. J Endourol 15:499-503

12. Vereczkei A, Feussner H, Negele T, Fritzsche F, Seitz T, Bubb H, Horvath OP (2004) Ergonomic assessment of the static stress confronted by surgeons during laparoscopic cholecystectomy. Surg Endosc 18:1118-1122

13. Herron DM, Gagner M, Kenyon TL, Swanstrom LL (2001) The minimally invasive surgical suite enters the 21 st century: a discussion of critical design elements. Surg Endosc 15:415-422 
14. Satava RM (2003) Disruptive visions: the operating room of the future. Surg Endosc 17:104-107

15. Hanna GB, Shimi SM, Cuschieri A (1998) Task performance in endoscopic surgery is influenced by location of the image display. Ann Surg 227:481-484

16. Seghers J, Jochem A, Spaepen A (2003) Posture, muscle activity, and muscle fatigue in prolonged VDT work at different screen height settings. Ergonomics 46:714-730

17. Middel B, Van Sonderen FLP (2002) Statistical significant change versus relevant or important change in (quasi) experimental design: some conceptual and methodological problems in estimating magnitude of intervention-related change in health services research. Int J Integrated Care 2:1-21

18. Cohen J (1988) Statistical power analysis for the behavioural sciences, 2nd edn. Academic Press, New York
19. Middel B, Stewart R, Bouma J, van Sonderen E, van den Heuvel WJA (2001) How to validate clinically important change in health-related functional status: is the magnitude of the effect size consistently related to magnitude of change as indicated by a global question rating? J Eval Clin Pract 7:399-410

20. Psihogios JP, Sommerich CM, Mirka GA, Moon SD (2001) A field evaluation of monitor placement effects in VDT users. Appl Ergon 32:313-325

21. Matern U, Faist M, Kehl K, Giebmeyer C, Buess G (2005) Monitor position in laparoscopic surgery. Surg Endosc 19: 436-440

22. Omar AM, Wade NJ, Brown SI, Cuschieri A (2005) Assessing the benefits of "gaze-down" display location in complex tasks. Surg Endosc 19:105-108 